\title{
Repeatability of small lung nodule measurement in low-dose lung screening: a phantom study
}

\author{
Yu Du, Gao-Feng Shi* ${ }^{*}$, Ya-Ning Wang, Qi Wang and Hui Feng
}

\begin{abstract}
Background: Lung cancer screening revealed that people with small pulmonary nodules are mostly asymptomatic and that some of these people are at risk of developing lung cancer, so we intended to explore the repeatability of small lung nodule measurement in low-dose lung screening.

Methods: We scanned eight ground-glass nodules (GGNs) and solid nodules, with diameters of 3, 5, 8, and $10 \mathrm{~mm}$. They were divided according to the different combination schemes of tube voltage (KV) and tube current (mA) as 70, 80, 100, and $120 \mathrm{KV}$, and currents of nine tubes were divided as 20, 30, 40, 50, 60, 70, 80, 90, and $100 \mathrm{mAs}$.

Results: Compared with the conventional dose group (120 kVp, $100 \mathrm{mAs})$, the nodule diameter and solid nodule volume measured by all scanning combinations were more consistent $(P>0.05)$, the volumes of $10 \mathrm{~mm}$ GGNs combinations were consistent $(P>0.05)$, the volumes of $8 \mathrm{~mm}$ GGNs were consistent $(P>0.05)$, the volumes of 5 $\mathrm{mm}$ GGNs combinations were consistent $(P>0.05)$, and the volumes of $3 \mathrm{~mm}$ were consistent $(P>0.05)$.

Conclusion: In lung cancer screening, CT parameters should be as follows: tube voltage is more than $80 \mathrm{kVp}$, and tube current is $80 \mathrm{mAs}$ in order to meet the requirements for the accurate measurement of the diameter and volume of pulmonary nodules.
\end{abstract}

Keywords: Computer tomography, Low dose, Pulmonary nodules, Body model, Lung cancer

\section{Background}

With the increasing popularity of lung cancer screening, the detection rate of small pulmonary nodules has increased. Lung cancer screening revealed that people with small pulmonary nodules are mostly asymptomatic and that some of these people are at risk of developing lung cancer [1]. Since the detection rate of small pulmonary nodules has increased, the subsequent problem is to determine how to deal with this as early as possible, and additional examination and treatment measures should be avoided. For malignant nodules, early diagnosis can provide a safer and clearer treatment plan. Considering

\footnotetext{
* Correspondence: shigaofeng123@yeah.net
}

Department of Radiology, the Fourth Hospital of Hebei Medical University, No. 12 Jiankang Rd. Changan District, Shijiazhuang 050011, China the possibility of false positives, the computed tomography $(\mathrm{CT})$ follow-up and monitoring of small nodules is very important. In addition, the possible radiation risk and economic cost of follow-up should also be comprehensively considered [2]. According to the International Lung Nodule Screening Guidelines, the size and growth rate of nodules are still well-recognized as important indicators to distinguish benign and malignant nodules [3, 4]. Compared with the nodule size in the first examination, the growth rate of the nodule can be calculated, in order to determine its benign and malignant nature. At present, the measurement of nodule size mainly includes diameter measurement, and the latest guidelines take volume measurement as a measurement standard $[4,5]$.

(c) The Author(s). 2020 Open Access This article is licensed under a Creative Commons Attribution 4.0 International License, which permits use, sharing, adaptation, distribution and reproduction in any medium or format, as long as you give appropriate credit to the original author(s) and the source, provide a link to the Creative Commons licence, and indicate if changes were made. The images or other third party material in this article are included in the article's Creative Commons licence, unless indicated otherwise in a credit line to the material. If material is not included in the article's Creative Commons licence and your intended use is not permitted by statutory regulation or exceeds the permitted use, you will need to obtain permission directly from the copyright holder. To view a copy of this licence, visit http://creativecommons.org/licenses/by/4.0/ The Creative Commons Public Domain Dedication waiver (http://creativecommons.org/publicdomain/zero/1.0/) applies to the data made available in this article, unless otherwise stated in a credit line to the data. 
In the lung cancer screening guidelines, such as the Lung Reporting and Data System (Lung-RADS) and the National Comprehensive Cancer Network (NCCN) guidelines, the mean diameter is used as the size standard for nodal follow-up and treatment [6, 7]. In the Dutch-Belgian Nelson test, volumes serve as a similar standard [5]. Indeed, the determination of the nodule follow-up and treatment plan during the lung cancer screening is not based on the actual size of the nodule in the surgical specimen, but on the size measured on the CT image and the changes before and after the followup [8]. Therefore, the present study focuses on the measurement accuracy of low-dose CT for small nodules and determines how to consistently measure the average diameter and volume, in order to determine its size and change, rather than the measurement accuracy of the actual nodule size.

The national lung screening test (NLST) revealed that the use of low-dose CT screening in high-risk groups could reduce lung cancer mortality $[9,10]$. According to the size and changes of nodules, lung cancer screening guidelines provide different treatment options. Therefore, in addition to the detection of pulmonary nodules, the repeatability of pulmonary nodule measurement is also an important factor in the follow-up and risk assessment of pulmonary nodules in CT screening. Since annual CT screening increases the risk of radiation-related cancer, the principle of minimizing the $\mathrm{CT}$ screening dose is also important [11]. Therefore, it is important to keep the accuracy of the screening image while reducing the radiation dose and avoiding large errors in the detection or measurement of pulmonary nodules.

In lung nodule screening, when using $120 \mathrm{kVp}$ of tube voltage, the tube current can be reduced to less than 100 $\mathrm{mAs}$, on the premise that the image quality can meet the diagnostic requirements. In some studies, the tube current was reduced to $80,70,60$, or even $10 \mathrm{mAs}$, and the radiation dose was reduced by $50-84 \%[12,13]$. Some studies have also reduced the tube current threshold to $20 \mathrm{mAs}$ for pulmonary nodules, including ground-glass nodules screening [14]. Another approach to reducing the radiation dose is to reduce the tube voltage. At present, the most common tube voltage is $100-140 \mathrm{kVp}$. However, some studies have considered that $80 \mathrm{kVp}$ is feasible for lung nodule screening [15]. Furthermore, few studies have concurrently reduced the tube current and tube voltage in carrying out the lung nodule screening. The phantom experiment is a very helpful method to avoid the extra radiation on patients. Therefore, the present study aimed to investigate the effect of different tube current and voltage combinations in low-dose scanning on the consistency of measurement of the pulmonary small nodule size using phantom. The conventional scanning dose $(120 \mathrm{kVp}, 100$ mAs) was used as the control group.

\section{Methods}

\section{Chest phantom}

The chest model used in the present study (Lungman, Kyoto Kagaku, Tokyo, Japan) was a model that could accurately simulate the human anatomy. The model has a size of $43 \times 40 \times 48 \mathrm{~cm}$ and was designed based on an adult male with a weight of $70 \mathrm{~kg}$. The body model was a male torso model with an artificial mediastinum and trachea, including the pulmonary vessels (right and left) and upper abdomen (diaphragm). The thickness of the chest wall was determined according to the clinical data. The X-ray absorptivity of the substitute material for simulating human soft tissue (polyurethane) and the simulated bone (epoxy resin) were both similar to that of the human tissue. The upper arm was in an abduction position to ensure that the trunk position is suitable for the CT examination. The use of this model can track the direction of the pulmonary vessels in space.

\section{Simulated pulmonary nodules}

For the simulated pulmonary nodules used in the present study, the solid nodules $(\mathrm{S},+100 \mathrm{HU})$ were made of polyurethane resin, and non-solid nodules (NS, $-800 \mathrm{HU}$ ) were made of polyurethane foam resin. In the present study, eight spherical simulated nodules with a smooth surface were used. The diameters were $3,5,8$, and $10 \mathrm{~mm}$, respectively, the volumes were $14.10,65.00$, 268.00 , and $523.00 \mathrm{~mm}^{3}$, respectively, and the CT attenuation values were $100 \mathrm{HU}$ and $-800 \mathrm{HU}$ (tube voltage: $120 \mathrm{kVp}$ ).

\section{Image acquisition}

A GE Revolution CT scanner [General Electric Co. (GE), USA] was used, and the combined scanning schemes of different tube voltages $(\mathrm{kV})$ and tube currents (MA) were adopted for the phantom. Combinations of tube voltage and tube currents were used. Four tube voltages (70, 80, 100, and $120 \mathrm{KV}$, respectively) and nine tube currents $(40,60,80,100,120,140,160,180$, and 200 $\mathrm{mA}$, respectively) were used. The CT scanning pitch was 0.992:1.000, and the rotation time of the rack was $0.5 \mathrm{~s}$. During the scanning, eight nodules were fixed on the vascular bundle in the phantom with double-sided adhesive tapes. The placement positions were the upper, middle, and lower lungs. The scans were separately performed, and six nodules could be placed for one scan. Each nodule and site were scanned three times, and these were placed in the left and right lungs, respectively. The scanning scope included the whole model from the thoracic entrance to the costophrenic angle. During the scanning process, it was ensured that the scope of each scan was the same. When collecting the images, the Stand and Bone algorithms were used to carry out the adaptive statistical iterative reconstruction 
(ASIR), in order to obtain the axial image, in which the ASIR ratio was $40 \%$, and both the slice thickness and interval of the reconstruction was $0.625 \mathrm{~mm}$.

\section{Measurement methods}

After the end of the scan, all images were imported into the Lung VCAR Single Lesion analysis software AW4.7 workstation (Advantage Workstation, GE, USA), and image processing was performed by a professional imaging physician (8 years of experience in chest imaging diagnosis). The software for pulmonary nodule analysis provided quantitative information on the pulmonary nodule size through volume segmentation for semi-automatic measurement. Apart from clicking again when the software system failed to segment the pulmonary nodules, a manual correction was not performed. The software calculated the diameter (anterior-posterior, left-right, and upper-lower diameters) and the volume of each pulmonary nodule, according to the lesion segmentation (Fig. 1). The average diameter obtained by calculating the average value of three diameter lines has been used in the Lung Cancer Screening Guidelines [16].

\section{Radiation dose}

The radiation dose parameters for the different scanning combinations were recorded: volume CT dose index $\left(C T D I_{v o l}\right)$ and dose length product (DLP). The unit of $\mathrm{CTDI}_{\mathrm{vol}}$ was $\mathrm{mGy} . \mathrm{DLP}=\mathrm{CTDI}_{\mathrm{vol}}(\mathrm{mGy}) \times$ scan length $(\mathrm{cm})$, and the unit was mGy.cm. An effective dose (ED) meant that the patient received an effective radiation dose during the examination. This was calculated using the following formula: $\mathrm{ED}=\mathrm{DLP} \times \mathrm{kn}$, in which the unit was $\mathrm{mSv}$, and $\mathrm{k}$ was the tissue weight factor. According to the European Union's "CT image quality standard guidelines," the appropriate weighted tissue factor of a standard chest is 0.017 . The CTDI ${ }_{\mathrm{vol}}$ and DLP of the different combinations were respectively recorded and calculated, and the ED was calculated.

\section{Statistics analysis}

All experimental data were statistically analyzed using Statistic Package for Social Science 21.0. Measurement data were expressed as mean \pm standard deviation $(\mathrm{x} \pm$ $\mathrm{SD})$. Count data were expressed in percentages (\%). The test of normality was conducted using W-tests. The homogeneity of variance was tested using F-tests. The multi-group comparison was conducted using a univariate analysis of variance. The backtesting was conducted using the least significant difference (LSD). Nonnormally distributed means of multiple samples or normally distributed means of multiple samples with a heterogeneity of variance were compared using nonparametric tests. Count data were compared using Chi-square tests. $P<0.05$ was considered statistically significant.

\section{Results}

Comparison of nodule diameters measured by different scanning combinations

In the present study, a total of 864 scans were completed. The results revealed that compared with the conventional dose group, the measured nodule diameters were in good agreement in all the other groups (all $P>$ 0.05 , Table 1 ), and the measured nodule diameters were in good agreement in all the other groups (all $P>0.05$, Table 2).

Also, compared with the conventional dose group, the measured nodule diameters were in good agreement in all the other groups (all $P>0.05$, Table 3 ), and the measured nodule diameters were in good agreement in all the other groups (all $P>0.05$, Table 4 ).

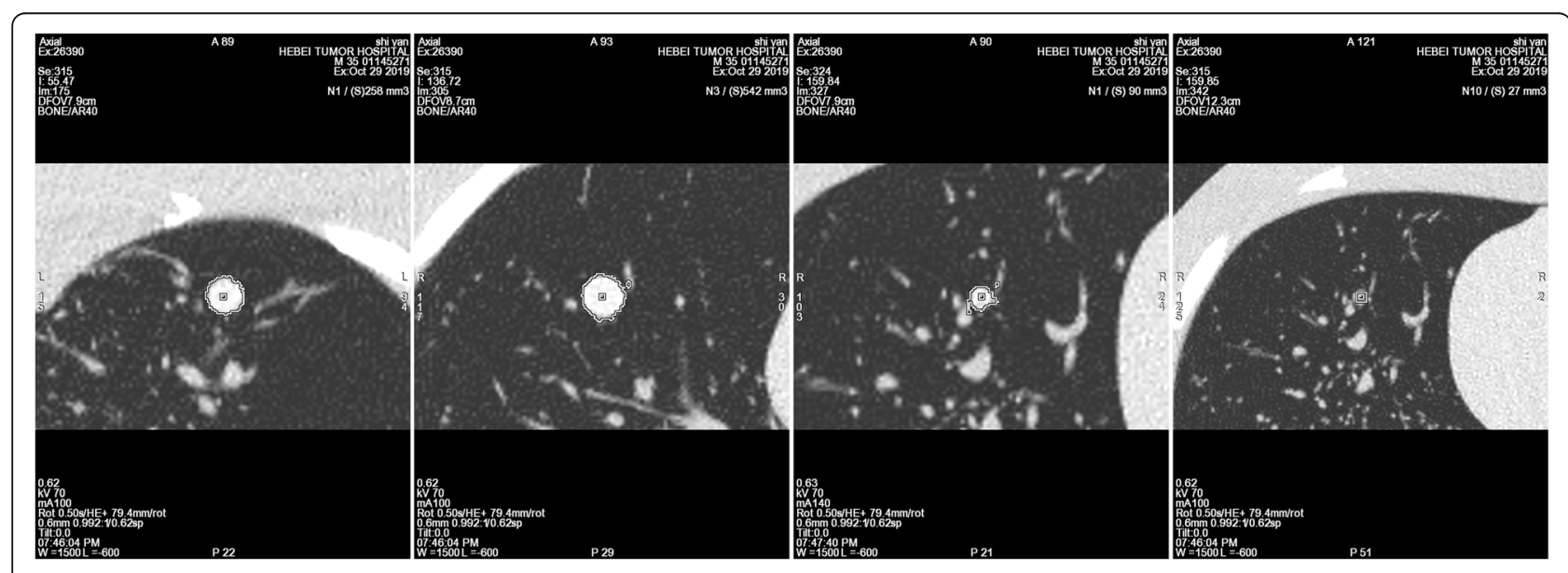

Fig. 1 CT images of pulmonary nodules of different sizes 
Table 1 Mean diameter of $10 \mathrm{~mm}$ nodules

\begin{tabular}{|c|c|c|c|c|c|c|c|}
\hline \multirow{3}{*}{ kV } & \multirow{3}{*}{$\mathrm{mAs}$} & \multicolumn{3}{|l|}{ Diameter } & \multicolumn{3}{|c|}{ Diameter } \\
\hline & & $10 \mathrm{~mm}$ & sd & $\mathbf{P}$ & $10 \mathrm{~mm}$ & sd & $\mathbf{P}$ \\
\hline & & GGN & & & $S$ & & \\
\hline 70 & 20 & 9.57 & 0.21 & 0.898 & 9.60 & 0.20 & 1.000 \\
\hline 70 & 30 & 10.90 & 0.61 & 0.837 & 9.60 & 0.20 & .830 \\
\hline 70 & 40 & 12.55 & 0.64 & 0.982 & 9.53 & 0.21 & .056 \\
\hline 70 & 50 & 9.33 & 0.15 & 0.985 & 10.37 & 0.86 & .079 \\
\hline 70 & 60 & 9.77 & 0.31 & 0.906 & 10.43 & 0.80 & .830 \\
\hline 70 & 70 & 10.80 & 0.44 & 0.939 & 9.67 & 0.12 & .747 \\
\hline 70 & 80 & 10.37 & 0.68 & 0.967 & 9.70 & 0.10 & 1.000 \\
\hline 70 & 90 & 10.00 & 0.26 & 0.951 & 9.60 & 0.20 & .200 \\
\hline 70 & 100 & 10.20 & 0.75 & 0.800 & 10.00 & 0.36 & .915 \\
\hline 80 & 20 & 12.20 & 0.89 & 0.953 & 9.63 & 0.15 & .592 \\
\hline 80 & 30 & 10.70 & 0.40 & 0.987 & 9.77 & 0.29 & .110 \\
\hline 80 & 40 & 9.73 & 0.50 & 0.901 & 10.10 & 0.46 & .392 \\
\hline 80 & 50 & 10.87 & 0.35 & 0.902 & 9.87 & 0.38 & .166 \\
\hline 80 & 60 & 10.67 & 0.47 & 0.951 & 10.03 & 0.59 & .285 \\
\hline 80 & 70 & 10.20 & 0.10 & 0.959 & 9.93 & 0.49 & .044 \\
\hline 80 & 80 & 10.10 & 0.46 & 0.944 & 10.23 & 0.71 & .285 \\
\hline 80 & 90 & 10.30 & 0.70 & 0.949 & 9.93 & 0.35 & .520 \\
\hline 80 & 100 & 10.23 & 0.87 & 0.974 & 9.80 & 0.26 & .453 \\
\hline 100 & 20 & 9.90 & 0.36 & 0.964 & 9.83 & 0.32 & .592 \\
\hline 100 & 30 & 10.03 & 0.38 & 0.990 & 9.77 & 0.15 & .520 \\
\hline 100 & 40 & 9.70 & 0.10 & 0.959 & 9.80 & 0.26 & .056 \\
\hline 100 & 50 & 10.10 & 0.44 & 0.949 & 10.20 & 0.87 & .520 \\
\hline 100 & 60 & 10.23 & 0.35 & 0.934 & 9.80 & 0.26 & .915 \\
\hline 100 & 70 & 10.43 & 0.31 & 0.944 & 9.63 & 0.15 & .392 \\
\hline 100 & 80 & 10.30 & 0.56 & 0.990 & 9.87 & 0.25 & .336 \\
\hline 100 & 90 & 9.70 & 0.10 & 0.969 & 9.90 & 0.30 & .915 \\
\hline 100 & 100 & 9.97 & 0.31 & 0.962 & 9.63 & 0.15 & .240 \\
\hline 120 & 20 & 10.07 & 0.42 & 0.918 & 9.97 & 0.32 & .453 \\
\hline 120 & 30 & 10.63 & 0.42 & 0.977 & 9.83 & 0.32 & .166 \\
\hline 120 & 40 & 9.87 & 0.38 & 0.918 & 10.03 & 0.40 & .453 \\
\hline 120 & 50 & 10.63 & 0.49 & 0.959 & 9.83 & 0.25 & .668 \\
\hline 120 & 60 & 10.10 & 0.40 & 0.982 & 9.73 & 0.15 & .747 \\
\hline 120 & 70 & 9.80 & 0.10 & 0.977 & 9.70 & 0.10 & .747 \\
\hline 120 & 80 & 9.87 & 0.21 & 0.972 & 9.70 & 0.10 & .747 \\
\hline 120 & 90 & 9.93 & 0.25 & 0.967 & 9.70 & 0.10 & .747 \\
\hline 120 & 100 & 10.00 & 0.26 & - & 9.70 & 0.10 & - \\
\hline
\end{tabular}

Comparison of nodule volumes measured by different scanning combinations

Compared with the conventional dose group, the difference between the combination of $80 \mathrm{kVp}$ and $50 \mathrm{mAs}$ and their combinations were not statistically significant in the $10-\mathrm{mm}$ NS group $(P>0.05)$. The measured nodule
Table 2 Mean diameter of $8 \mathrm{~mm}$ nodules

\begin{tabular}{|c|c|c|c|c|c|c|c|}
\hline \multirow[b]{2}{*}{ kV } & \multirow[b]{2}{*}{ mAs } & \multicolumn{3}{|l|}{ Diameter } & \multicolumn{3}{|c|}{ Diameter } \\
\hline & & $\begin{array}{l}8 \mathrm{~mm} \text { sd } \\
\text { GGN }\end{array}$ & sd & $P$ & $\begin{array}{l}8 \mathrm{~mm} \\
\mathrm{~S}\end{array}$ & sd & $P$ \\
\hline 70 & 20 & 9.10 & 0.10 & .070 & 8.10 & 0.70 & .068 \\
\hline 70 & 30 & 8.53 & 0.91 & .068 & 8.83 & 0.74 & .058 \\
\hline 70 & 40 & 8.47 & 0.95 & .857 & 8.00 & 1.41 & .078 \\
\hline 70 & 50 & 7.97 & 0.25 & .418 & 8.20 & 0.56 & .058 \\
\hline 70 & 60 & 8.70 & 0.75 & .753 & 8.83 & 0.31 & .365 \\
\hline 70 & 70 & 7.87 & 0.35 & .140 & 8.50 & 0.56 & .192 \\
\hline 70 & 80 & 8.20 & 0.40 & .787 & 8.63 & 0.72 & .420 \\
\hline 70 & 90 & 7.90 & 0.30 & .892 & 7.93 & 0.15 & .420 \\
\hline 70 & 100 & 8.00 & 0.20 & .027 & 7.93 & 0.25 & .091 \\
\hline 80 & 20 & 8.77 & 1.46 & .033 & 8.33 & 0.12 & .840 \\
\hline 80 & 30 & 8.70 & 0.10 & .964 & 8.13 & 0.40 & .420 \\
\hline 80 & 40 & 8.07 & 0.15 & .964 & 7.93 & 0.15 & .762 \\
\hline 80 & 50 & 8.07 & 0.15 & .964 & 8.10 & 0.40 & .687 \\
\hline 80 & 60 & 8.13 & 0.21 & .097 & 8.07 & 0.38 & .097 \\
\hline 80 & 70 & 8.77 & 0.15 & .080 & 8.90 & 0.10 & .545 \\
\hline 80 & 80 & 8.73 & 0.15 & 1.000 & 8.00 & 0.26 & .614 \\
\hline 80 & 90 & 8.10 & 0.20 & .857 & 8.03 & 0.31 & .481 \\
\hline 80 & 100 & 7.97 & 0.12 & .210 & 7.97 & 0.21 & .269 \\
\hline 100 & 20 & 9.03 & 0.80 & .118 & 7.83 & 0.25 & .762 \\
\hline 100 & 30 & 9.07 & 0.67 & .262 & 8.10 & 0.20 & .365 \\
\hline 100 & 40 & 8.93 & 1.36 & .323 & 7.90 & 0.20 & .315 \\
\hline 100 & 50 & 8.83 & 0.75 & .822 & 7.87 & 0.35 & .481 \\
\hline 100 & 60 & 7.93 & 0.25 & .822 & 7.97 & 0.12 & .365 \\
\hline 100 & 70 & 7.93 & 0.15 & .964 & 7.90 & 0.10 & .420 \\
\hline 100 & 80 & 8.13 & 0.21 & .857 & 7.93 & 0.15 & .365 \\
\hline 100 & 90 & 7.97 & 0.21 & .369 & 7.90 & 0.26 & .687 \\
\hline 100 & 100 & 8.77 & 0.74 & .822 & 8.07 & 0.15 & .315 \\
\hline 120 & 20 & 7.93 & 0.15 & 1.000 & 7.87 & 0.35 & .420 \\
\hline 120 & 30 & 8.10 & 0.20 & .822 & 7.93 & 0.15 & .315 \\
\hline 120 & 40 & 7.93 & 0.15 & .892 & 7.87 & 0.21 & .481 \\
\hline 120 & 50 & 8.00 & 0.26 & .928 & 8.43 & 0.21 & .420 \\
\hline 120 & 60 & 8.17 & 0.15 & .472 & 7.93 & 0.15 & .269 \\
\hline 120 & 70 & 8.63 & 0.12 & .892 & 7.83 & 0.06 & .420 \\
\hline 120 & 80 & 8.00 & 0.10 & .822 & 7.93 & 0.25 & .614 \\
\hline 120 & 90 & 7.93 & 0.06 & .857 & 8.03 & 0.12 & .133 \\
\hline 120 & 100 & 7.97 & 0.06 & - & 7.70 & 0.20 & - \\
\hline
\end{tabular}

volumes were in good agreement between the combination of $70 \mathrm{kVp}$ and $20 \mathrm{mAs}$, and the above combinations in the $10-\mathrm{mm} \mathrm{S}$ group (all $P>0.05$, Table 5).

Compared with the conventional dose group, the difference between the combination of $80 \mathrm{kVp}$ and $50 \mathrm{mAs}$ and the above combinations was not statistically 
Table 3 Mean diameter of $5 \mathrm{~mm}$ nodules

\begin{tabular}{|c|c|c|c|c|c|c|c|}
\hline \multirow[b]{2}{*}{ kV } & \multirow[b]{2}{*}{ mAs } & \multicolumn{3}{|l|}{ Diameter } & \multicolumn{3}{|c|}{ Diameter } \\
\hline & & $\begin{array}{l}5 \mathrm{~mm} \\
\mathrm{GGN}\end{array}$ & sd & $P$ & $\begin{array}{l}5 \mathrm{~mm} \\
\mathrm{~S}\end{array}$ & sd & $\mathbf{P}$ \\
\hline 70 & 20 & 5.27 & 0.38 & .061 & 5.07 & 0.06 & 1.000 \\
\hline 70 & 30 & 4.67 & 0.67 & .294 & 5.07 & 0.06 & 1.000 \\
\hline 70 & 40 & 4.93 & 0.21 & .143 & 5.07 & 0.06 & 1.000 \\
\hline 70 & 50 & 4.80 & 0.44 & .344 & 5.07 & 0.06 & .471 \\
\hline 70 & 60 & 5.57 & 0.31 & .916 & 5.17 & 0.12 & 1.000 \\
\hline 70 & 70 & 5.23 & 0.61 & .294 & 5.07 & 0.06 & 1.000 \\
\hline 70 & 80 & 4.93 & 0.21 & .294 & 5.07 & 0.06 & 1.000 \\
\hline 70 & 90 & 4.93 & 0.21 & .143 & 5.07 & 0.06 & 1.000 \\
\hline 70 & 100 & 4.80 & 0.44 & .095 & 5.07 & 0.06 & .151 \\
\hline 80 & 20 & 4.73 & 0.35 & .143 & 4.87 & 0.40 & 1.000 \\
\hline 80 & 30 & 4.80 & 0.44 & .294 & 5.07 & 0.06 & 1.000 \\
\hline 80 & 40 & 4.93 & 0.21 & .294 & 5.07 & 0.06 & 1.000 \\
\hline 80 & 50 & 4.93 & 0.21 & .294 & 5.07 & 0.06 & 1.000 \\
\hline 80 & 60 & 4.93 & 0.21 & .673 & 5.07 & 0.06 & 1.000 \\
\hline 80 & 70 & 5.13 & 0.67 & .294 & 5.07 & 0.06 & 1.000 \\
\hline 80 & 80 & 4.93 & 0.21 & .294 & 5.07 & 0.06 & 1.000 \\
\hline 80 & 90 & 4.93 & 0.21 & .294 & 5.07 & 0.06 & 1.000 \\
\hline 80 & 100 & 4.93 & 0.21 & .143 & 5.07 & 0.06 & 1.000 \\
\hline 100 & 20 & 4.80 & 0.44 & .294 & 5.07 & 0.06 & 1.000 \\
\hline 100 & 30 & 4.93 & 0.21 & .143 & 5.07 & 0.06 & 1.000 \\
\hline 100 & 40 & 4.80 & 0.44 & .117 & 5.07 & 0.06 & 1.000 \\
\hline 100 & 50 & 5.77 & 0.76 & .248 & 5.07 & 0.06 & 1.000 \\
\hline 100 & 60 & 5.63 & 0.51 & .143 & 5.07 & 0.06 & 1.000 \\
\hline 100 & 70 & 4.80 & 0.44 & .294 & 5.07 & 0.06 & .151 \\
\hline 100 & 80 & 4.93 & 0.21 & .294 & 4.87 & 0.40 & 1.000 \\
\hline 100 & 90 & 4.93 & 0.21 & .294 & 5.07 & 0.06 & 1.000 \\
\hline 100 & 100 & 4.93 & 0.21 & .173 & 5.07 & 0.06 & 1.000 \\
\hline 120 & 20 & 5.70 & 0.87 & .294 & 5.07 & 0.06 & 1.000 \\
\hline 120 & 30 & 4.93 & 0.21 & .095 & 5.07 & 0.06 & .151 \\
\hline 120 & 40 & 4.73 & 0.35 & .248 & 4.87 & 0.40 & .000 \\
\hline 120 & 50 & 4.90 & 0.20 & .344 & 5.83 & 0.64 & 1.000 \\
\hline 120 & 60 & 4.97 & 0.15 & .143 & 5.07 & 0.06 & .630 \\
\hline 120 & 70 & 4.80 & 0.26 & .294 & 5.00 & 0.10 & 1.000 \\
\hline 120 & 80 & 4.93 & 0.21 & .294 & 5.07 & 0.06 & 1.000 \\
\hline 120 & 90 & 4.93 & 0.21 & .344 & 5.07 & 0.06 & .471 \\
\hline 120 & 100 & 4.97 & 0.23 & - & 5.17 & 0.12 & - \\
\hline
\end{tabular}

significant in the 8 -mm NS group $(P>0.05)$. The measured nodule volumes were in good agreement between the combination of $70 \mathrm{kVp}$ and $20 \mathrm{mAs}$, and the above combinations in the $8-\mathrm{mm} \mathrm{S}$ group (all $P>0.05$, Table 6).

Compared with the conventional dose group, the difference between the combination of $80 \mathrm{kVp}$ and
Table 4 Mean diameter of $3 \mathrm{~mm}$ nodules

\begin{tabular}{|c|c|c|c|c|c|c|c|}
\hline & & Diameter & & & Diameter & & \\
\hline kV & mAs & 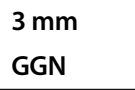 & sd & $\mathbf{P}$ & $\begin{array}{l}3 \mathrm{~mm} \\
\mathrm{~s}\end{array}$ & sd & $\mathbf{P}$ \\
\hline 70 & 20 & 3.80 & 0.87 & .062 & 3.23 & 0.23 & .202 \\
\hline 70 & 30 & 4.53 & 0.67 & .028 & 2.87 & 0.21 & .202 \\
\hline 70 & 40 & 4.67 & 1.12 & .034 & 2.87 & 0.21 & .352 \\
\hline 70 & 50 & 4.63 & 0.50 & .074 & 2.97 & 0.23 & .202 \\
\hline 70 & 60 & 3.10 & 0.40 & .042 & 3.60 & 0.26 & .352 \\
\hline 70 & 70 & 3.00 & 0.44 & .074 & 3.50 & 0.40 & 1.000 \\
\hline 70 & 80 & 3.10 & 0.40 & .089 & 3.23 & 0.23 & .641 \\
\hline 70 & 90 & 3.13 & 0.35 & .042 & 3.10 & 0.40 & .907 \\
\hline 70 & 100 & 3.00 & 0.44 & .042 & 3.20 & 0.44 & .641 \\
\hline 80 & 20 & 3.00 & 0.44 & .391 & 3.37 & 0.23 & .001 \\
\hline 80 & 30 & 4.13 & 0.29 & .074 & 4.20 & 0.62 & .641 \\
\hline 80 & 40 & 3.10 & 0.40 & .042 & 3.10 & 0.40 & .641 \\
\hline 80 & 50 & 3.00 & 0.44 & .042 & 3.10 & 0.40 & .352 \\
\hline 80 & 60 & 3.00 & 0.44 & .265 & 2.97 & 0.23 & .641 \\
\hline 80 & 70 & 3.37 & 0.23 & .074 & 3.37 & 0.23 & .641 \\
\hline 80 & 80 & 3.10 & 0.40 & .042 & 3.10 & 0.40 & .641 \\
\hline 80 & 90 & 3.00 & 0.44 & .074 & 3.10 & 0.40 & .415 \\
\hline 80 & 100 & 3.10 & 0.40 & .051 & 3.00 & 0.44 & .641 \\
\hline 100 & 20 & 3.03 & 0.50 & .089 & 3.10 & 0.40 & 1.000 \\
\hline 100 & 30 & 3.13 & 0.35 & .023 & 3.23 & 0.23 & .641 \\
\hline 100 & 40 & 2.90 & 0.53 & .023 & 3.10 & 0.40 & .352 \\
\hline 100 & 50 & 2.90 & 0.53 & .074 & 2.97 & 0.23 & .641 \\
\hline 100 & 60 & 3.10 & 0.40 & .074 & 3.10 & 0.40 & .202 \\
\hline 100 & 70 & 3.10 & 0.40 & .074 & 2.87 & 0.21 & .641 \\
\hline 100 & 80 & 3.10 & 0.40 & .147 & 3.10 & 0.40 & .202 \\
\hline 100 & 90 & 3.23 & 0.23 & .147 & 2.87 & 0.21 & 1.000 \\
\hline 100 & 100 & 3.23 & 0.23 & .147 & 3.23 & 0.23 & .641 \\
\hline 120 & 20 & 3.23 & 0.23 & .074 & 3.10 & 0.40 & .641 \\
\hline 120 & 30 & 3.10 & 0.40 & .023 & 3.10 & 0.40 & .641 \\
\hline 120 & 40 & 2.90 & 0.53 & .074 & 3.10 & 0.40 & .726 \\
\hline 120 & 50 & 3.10 & 0.40 & .863 & 3.33 & 0.21 & .641 \\
\hline 120 & 60 & 3.73 & 0.59 & .074 & 3.10 & 0.40 & .815 \\
\hline 120 & 70 & 3.10 & 0.40 & .074 & 3.17 & 0.35 & .415 \\
\hline 120 & 80 & 3.10 & 0.40 & .074 & 3.00 & 0.44 & .726 \\
\hline 120 & 90 & 3.10 & 0.40 & .074 & 3.13 & 0.35 & .641 \\
\hline 120 & 100 & 3.10 & 0.40 & - & 3.10 & 0.40 & - \\
\hline
\end{tabular}

$50 \mathrm{mAs}$ and the above combinations was not statistically significant in the $5-\mathrm{mm}$ NS group $(P>0.05)$. The measured nodule volumes were in good agreement between the combination of $70 \mathrm{kVp}$ and 20 $\mathrm{mAs}$, and the above combinations in the $5-\mathrm{mm} \mathrm{S}$ group (all $P>0.05$, Table 7 ). 
Table 5 the volume of $10 \mathrm{~mm}$ nodules

\begin{tabular}{|c|c|c|c|c|c|c|c|}
\hline \multirow[b]{2}{*}{ kV } & \multirow[b]{2}{*}{$\mathrm{mAs}$} & \multicolumn{3}{|l|}{ Volume } & \multicolumn{3}{|l|}{ Volume } \\
\hline & & $\begin{array}{l}10 \mathrm{~mm} \\
\mathrm{GGN}\end{array}$ & sd & $\mathbf{P}$ & $\begin{array}{l}10 \mathrm{~mm} \\
\mathrm{~S}\end{array}$ & sd & $\mathbf{P}$ \\
\hline 70 & 20 & 293.00 & 48.75 & .000 & 495.00 & 13.23 & .662 \\
\hline 70 & 30 & 343.00 & 37.32 & .000 & 492.33 & 8.39 & \\
\hline 70 & 40 & 386.33 & 37.02 & .015 & 511.33 & 10.02 & .00 \\
\hline 70 & 50 & 371.67 & 11.06 & .840 & 535.67 & 26.27 & .000 \\
\hline 70 & 60 & 345.33 & 6.66 & .003 & 521.33 & 17.56 & .027 \\
\hline 70 & 70 & 378.33 & 10.97 & .000 & 508.67 & 2.89 & .00 \\
\hline 70 & 80 & 387.00 & 5.57 & .000 & 515.33 & 6.66 & .016 \\
\hline 70 & 90 & 411.33 & 6.03 & .000 & 510.00 & 2.65 & \\
\hline 70 & 100 & 402.00 & 3.00 & .228 & 510.33 & 1.53 & \\
\hline 80 & 20 & 357.00 & 17.06 & .000 & 498.33 & 3.21 & .12 \\
\hline 80 & 30 & 408.67 & 4.04 & .357 & 504.33 & 3.06 & \\
\hline 80 & 40 & 353.67 & 2.52 & .000 & 505.33 & 1.53 & \\
\hline 80 & 50 & 521.00 & 5.29 & .000 & 505.33 & 2.08 & .129 \\
\hline 80 & 60 & 460.00 & 5.29 & .000 & 504.33 & 2.08 & .158 \\
\hline 80 & 70 & 424.67 & 1.53 & .000 & 503.67 & 2.08 & \\
\hline 80 & 80 & 434.33 & 8.02 & .000 & 506.00 & 6.56 & .007 \\
\hline 80 & 90 & 460.00 & 7.55 & .000 & 512.00 & 8.19 & .003 \\
\hline 80 & 100 & 496.00 & 13.11 & .000 & 513.67 & 7.64 & .093 \\
\hline 100 & 20 & 393.33 & 7.09 & .000 & 505.33 & 5.69 & .002 \\
\hline 100 & 30 & 407.33 & 15.18 & .000 & 514.33 & 5.86 & .093 \\
\hline 100 & 40 & 425.67 & 5.86 & .000 & 505.33 & 6.11 & .001 \\
\hline 100 & 50 & 437.00 & 2.00 & .000 & 515.67 & 5.51 & .031 \\
\hline 100 & 60 & 462.67 & 6.11 & .000 & 508.33 & 3.51 & .036 \\
\hline 100 & 70 & 472.00 & 7.00 & .000 & 508.00 & 2.65 & .007 \\
\hline 100 & 80 & 488.33 & 3.21 & .000 & 512.00 & 5.29 & \\
\hline 100 & 90 & 477.00 & 3.61 & .000 & 505.00 & 6.00 & .014 \\
\hline 100 & 100 & 506.67 & 4.73 & .000 & 510.33 & 1.53 & \\
\hline 120 & 20 & 416.33 & 5.13 & .000 & 512.00 & 3.61 & .008 \\
\hline 120 & 30 & 449.00 & 17.78 & .000 & 511.67 & 5.03 & 000 \\
\hline 120 & 40 & 430.33 & 5.86 & .000 & 517.33 & 6.11 & .007 \\
\hline 120 & 50 & 463.33 & 4.51 & .000 & 512.00 & 4.00 & .104 \\
\hline 120 & 60 & 490.33 & 4.16 & .000 & 505.00 & 4.58 & .211 \\
\hline 120 & 70 & 507.33 & 7.02 & .000 & 502.67 & 3.21 & .009 \\
\hline 120 & 80 & 499.33 & 3.51 & .000 & 511.33 & 2.08 & .083 \\
\hline 120 & 90 & 497.33 & 3.21 & .000 & 505.67 & 4.73 & .008 \\
\hline 120 & 100 & 502.33 & 6.51 & & 511.67 & 3.21 & \\
\hline
\end{tabular}

Compared with the conventional dose group, the measured nodule volumes were in good agreement between the combination of $80 \mathrm{kVp}$ and $80 \mathrm{mAs}$, and the above combinations in the $10-\mathrm{mmS}$ group (all $P>0.05)$. The measured nodule volumes were in good agreement between the combination of $70 \mathrm{kVp}$ and
Table 6 the volume of $8 \mathrm{~mm}$ nodules

\begin{tabular}{|c|c|c|c|c|c|c|c|}
\hline \multirow{3}{*}{ kV } & \multirow{3}{*}{ mAs } & \multicolumn{3}{|c|}{ Volume } & \multicolumn{3}{|c|}{ Volume } \\
\hline & & $8 \mathrm{~mm}$ & sd & $\mathbf{P}$ & $8 \mathrm{~mm}$ & sd & $\mathbf{P}$ \\
\hline & & GGN & & & $S$ & & \\
\hline 70 & 20 & 183.67 & 25.42 & .001 & 255.00 & 1.00 & .569 \\
\hline 70 & 30 & 202.67 & 9.45 & .026 & 252.33 & 6.43 & .320 \\
\hline 70 & 40 & 196.67 & 7.64 & .015 & 250.33 & 7.02 & .137 \\
\hline 70 & 50 & 198.00 & 6.56 & .000 & 248.00 & 13.08 & .137 \\
\hline 70 & 60 & 227.33 & 15.63 & .001 & 262.00 & 9.54 & .887 \\
\hline 70 & 70 & 203.67 & 2.08 & .000 & 255.67 & 4.04 & .393 \\
\hline 70 & 80 & 239.00 & 3.00 & .000 & 251.00 & 7.21 & .000 \\
\hline 70 & 90 & 216.00 & 3.61 & .298 & 238.00 & 5.29 & .042 \\
\hline 70 & 100 & 189.67 & 5.03 & .030 & 245.33 & 9.50 & .434 \\
\hline 80 & 20 & 196.33 & 7.09 & .004 & 258.67 & 4.04 & .010 \\
\hline 80 & 30 & 200.67 & 3.06 & .013 & 242.67 & 6.66 & .078 \\
\hline 80 & 40 & 198.33 & 4.16 & .000 & 246.67 & 6.81 & .007 \\
\hline 80 & 50 & 209.00 & 2.00 & .000 & 242.00 & 2.65 & .669 \\
\hline 80 & 60 & 218.33 & 3.51 & .000 & 253.00 & 3.61 & .049 \\
\hline 80 & 70 & 261.33 & 2.08 & .000 & 264.33 & 1.53 & .943 \\
\hline 80 & 80 & 268.00 & 4.00 & .000 & 255.33 & 5.86 & .776 \\
\hline 80 & 90 & 244.00 & 7.21 & .000 & 256.33 & 6.11 & .569 \\
\hline 80 & 100 & 228.00 & 5.57 & .000 & 257.67 & 7.51 & .067 \\
\hline 100 & 20 & 217.67 & 3.06 & .000 & 246.33 & 4.16 & .042 \\
\hline 100 & 30 & 235.67 & 5.03 & .000 & 245.33 & 5.13 & .049 \\
\hline 100 & 40 & 236.33 & 1.53 & .000 & 245.67 & 6.66 & .057 \\
\hline 100 & 50 & 261.33 & 2.08 & .000 & 246.00 & 7.94 & .202 \\
\hline 100 & 60 & 250.00 & 12.77 & .000 & 249.00 & 4.00 & .256 \\
\hline 100 & 70 & 226.67 & 3.79 & .000 & 249.67 & 4.16 & .355 \\
\hline 100 & 80 & 235.33 & 3.21 & .000 & 250.67 & 1.53 & .887 \\
\hline 100 & 90 & 266.67 & 3.06 & .000 & 254.33 & 4.73 & 1.000 \\
\hline 100 & 100 & 316.00 & 4.58 & .326 & 255.00 & 3.61 & .434 \\
\hline 120 & 20 & 189.33 & 4.93 & .000 & 251.33 & 4.93 & .943 \\
\hline 120 & 30 & 237.33 & 4.73 & .000 & 254.67 & 4.04 & .104 \\
\hline 120 & 40 & 227.67 & 8.08 & .000 & 247.33 & 2.08 & .042 \\
\hline 120 & 50 & 262.67 & 3.21 & .000 & 264.67 & 6.66 & .202 \\
\hline 120 & 60 & 255.67 & 2.89 & .000 & 249.00 & 2.00 & .522 \\
\hline 120 & 70 & 282.67 & 4.04 & .000 & 252.00 & 4.00 & .569 \\
\hline 120 & 80 & 250.33 & 2.52 & .000 & 252.33 & 4.16 & .887 \\
\hline 120 & 90 & 255.00 & 2.00 & .000 & 254.33 & 4.73 & .831 \\
\hline 120 & 100 & 254.00 & 5.00 & - & 254.00 & 1.73 & \\
\hline
\end{tabular}

$20 \mathrm{mAs}$, and the above combinations in the $3-\mathrm{mm} \mathrm{S}$ group (all $P>0.05$, Table 8 ).

\section{Discussion}

The results of the present study revealed that compared with the conventional dose group (120 kVp and $100 \mathrm{mAs})$, 
Table 7 the volume of $5 \mathrm{~mm}$ nodules

\begin{tabular}{|c|c|c|c|c|c|c|c|}
\hline \multirow[b]{2}{*}{ kV } & \multirow[b]{2}{*}{ mAs } & \multicolumn{3}{|c|}{ Volume } & \multicolumn{3}{|c|}{ Volume } \\
\hline & & $\begin{array}{l}5 \mathrm{~mm} \\
\text { GGN }\end{array}$ & sd & $P$ & $\begin{array}{l}5 \mathrm{~mm} \\
\mathrm{~S}\end{array}$ & sd & $\mathbf{P}$ \\
\hline 70 & 20 & 33.67 & 1.53 & .709 & 60.67 & 4.51 & .059 \\
\hline 70 & 30 & 34.33 & 3.06 & .001 & 63.67 & 1.53 & .022 \\
\hline 70 & 40 & 40.00 & 2.00 & .576 & 64.33 & 1.53 & .396 \\
\hline 70 & 50 & 34.67 & 2.52 & .000 & 62.00 & 2.00 & \\
\hline 70 & 60 & 43.33 & 2.52 & .000 & 62.67 & 2.08 & .0 \\
\hline 70 & 70 & 43.67 & 1.53 & .000 & 64.00 & 2.00 & \\
\hline 70 & 80 & 44.00 & 1.00 & .000 & 65.33 & 1.53 & .013 \\
\hline 70 & 90 & 43.67 & 2.08 & .000 & 64.67 & 1.53 & \\
\hline 70 & 100 & 44.33 & 2.08 & .004 & 64.00 & 2.00 & .671 \\
\hline 80 & 20 & 39.00 & 3.61 & .138 & 60.00 & 1.00 & .26 \\
\hline 80 & 30 & 36.33 & 1.53 & .028 & 62.67 & 2.08 & .139 \\
\hline 80 & 40 & 37.67 & 1.53 & .000 & 63.00 & 1.00 & .092 \\
\hline 80 & 50 & 42.00 & 1.00 & .000 & 63.33 & 1.53 & .036 \\
\hline 80 & 60 & 45.00 & 1.00 & .000 & 64.00 & 1.00 & .059 \\
\hline 80 & 70 & 53.00 & 1.00 & .000 & 63.67 & 1.53 & \\
\hline 80 & 80 & 49.00 & 6.56 & .000 & 66.67 & 1.53 & .000 \\
\hline 80 & 90 & 47.33 & 1.53 & .000 & 67.00 & 2.65 & .000 \\
\hline 80 & 100 & 48.67 & 2.08 & .000 & 69.00 & 3.61 & .013 \\
\hline 100 & 20 & 43.67 & 1.53 & .000 & 64.67 & 1.53 & $.00^{\top}$ \\
\hline 100 & 30 & 44.33 & 1.53 & .000 & 66.33 & 2.08 & .000 \\
\hline 100 & 40 & 46.33 & 1.53 & .000 & 66.67 & 2.08 & .036 \\
\hline 100 & 50 & 49.00 & 3.61 & .000 & 64.00 & 3.61 & \\
\hline 100 & 60 & 45.67 & 3.51 & .000 & 65.33 & 2.08 & .000 \\
\hline 100 & 70 & 46.67 & 1.53 & .000 & 67.33 & 2.08 & .092 \\
\hline 100 & 80 & 48.00 & 1.00 & .000 & 63.33 & 1.53 & .000 \\
\hline 100 & 90 & 56.33 & 1.53 & .000 & 66.67 & 1.53 & .000 \\
\hline 100 & 100 & 57.67 & 0.58 & .000 & 66.67 & 0.58 & .002 \\
\hline 120 & 20 & 51.67 & 0.58 & .000 & 65.67 & 1.15 & .002 \\
\hline 120 & 30 & 44.67 & 0.58 & .000 & 65.67 & 1.53 & .524 \\
\hline 120 & 40 & 45.33 & 1.15 & .000 & 61.67 & 1.15 & .209 \\
\hline 120 & 50 & 55.67 & 2.08 & .000 & 62.33 & 0.58 & 000 \\
\hline 120 & 60 & 56.33 & 2.52 & .000 & 67.33 & 0.58 & .001 \\
\hline 120 & 70 & 56.33 & 0.58 & .000 & 66.00 & 1.00 & .000 \\
\hline 120 & 80 & 57.00 & 1.73 & .000 & 67.67 & 0.58 & .000 \\
\hline 120 & 90 & 56.33 & 2.08 & .000 & 66.67 & 1.53 & .001 \\
\hline 120 & 100 & 55.67 & 1.15 & & 66.33 & 2.08 & \\
\hline
\end{tabular}

the measured nodule diameters were in good agreement in all scanning combination groups, but the differences were not all statistically significant. The measured nodule volumes were in good agreement between all scanning combination groups and the conventional dose group, but the differences were not all statistically significant.
Table 8 the volume of $3 \mathrm{~mm}$ nodules

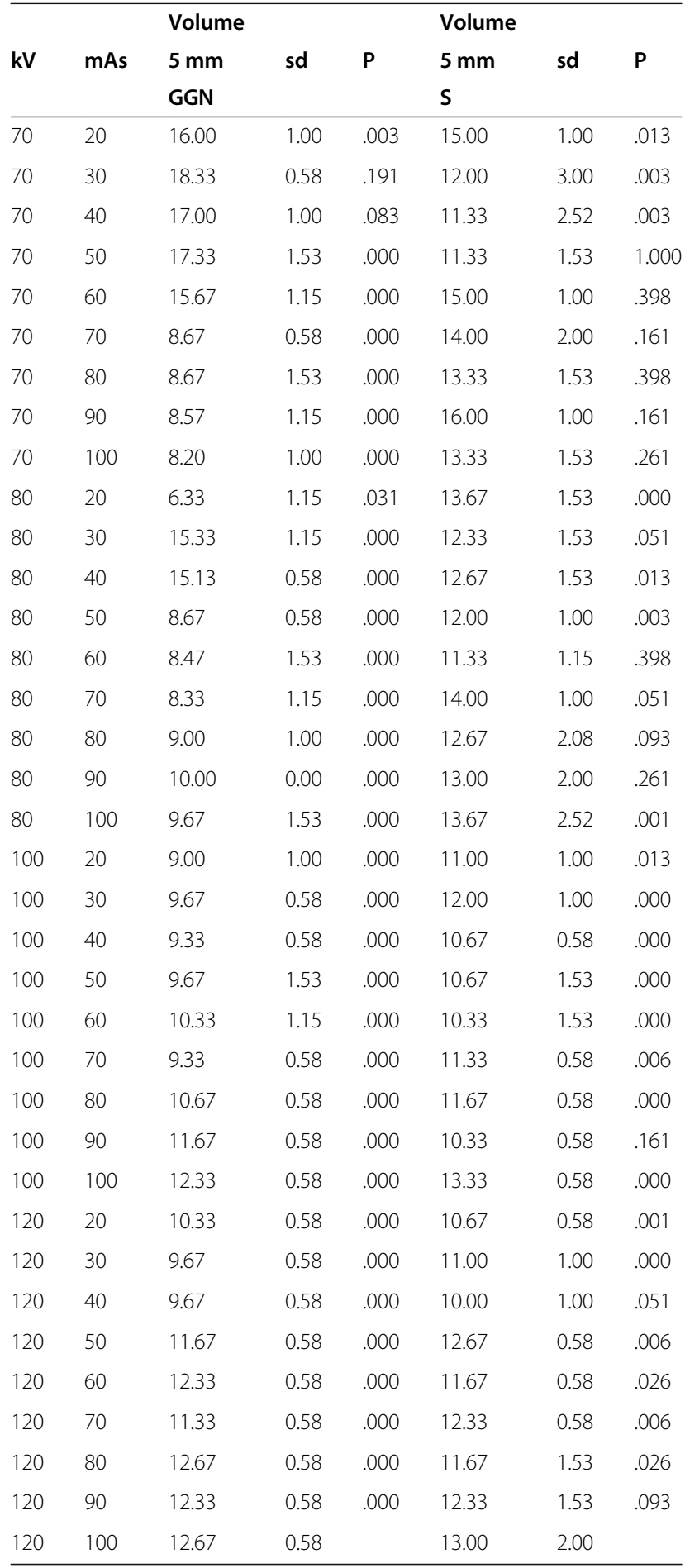

Different scanning doses can be obtained by changing the combination of tube voltage and tube current. In the present study, the lowest scanning dose $(70 \mathrm{kVp}$ and 20 $\mathrm{mAs}$ ) was $0.17 \mathrm{mSv}$, which was only $3.98 \%$ of the conventional dose (120 kVp and $100 \mathrm{mAs} ; 4.24 \mathrm{mSv})$. For solid and ground-glass small nodules, the difference in 
the mean diameter of nodules measured by various scanning doses was not statistically significant, the measurement result of the lower scanning dose was in good agreement with that of the conventional dose, and the results revealed that the decrease in scanning dose in a certain range has little impact on the measurement of the mean diameter of nodules.

Compared with the measurement of the nodule diameter, changes in nodule volumes measured by different scanning combinations were relatively complex. For solid nodules with different diameters, even with a lower scanning dose, the results were consistent. For 10-mm ground-glass nodules, better consistency could be obtained by using the scanning combination of more than $80 \mathrm{kVp}$ and $50 \mathrm{mAs}$. For $8-\mathrm{mm}$ and 5-mm ground-glass nodules, better consistency could be obtained by using the scanning combination of more than $80 \mathrm{kVp}$ and 70 mAs. For 3-mm ground-glass nodules, better consistency could be obtained by using the scanning combination of more than $80 \mathrm{kVp}$ and $80 \mathrm{mAs}$. With the decrease in scanning dose, the signal-to-noise ratio (SNR) also decreased. In the present study, the segmentation and volume measurement of ground-glass nodules using the pulmonary nodule analysis software was significantly affected, with a decrease in nodule diameter, and this effect was more obvious. Therefore, better consistency could only be obtained by using the scanning combinations of higher tube voltage and tube current. The reason may be because as the tube voltage and tube current decreased, the software had more difficulty accurately segmenting the boundary of the ground-glass nodules. In particular, this was difficult to distinguish from the surrounding vascular structure, resulting in significant differences in volume measurement results. The scanning dose of the combination of $100 \mathrm{kVp}$ and $20 \mathrm{mAs}$ was $0.53 \mathrm{mSv}$, while the scanning dose of the combination of $80 \mathrm{kVp}$ and $40 \mathrm{mAs}$ was $0.54 \mathrm{mSv}$. The scanning doses of these two combinations were similar. However, the consistency of the measurement results of the latter to the ground-glass nodule volume was poor. This suggests that compared with the reduction in tube current, the effect of reducing the tube voltage on the measurement of the volume of ground-glass nodules may be greater.

The present study has the following limitations. First, in the present study, the phantom was used for the experiment. Therefore, the conclusion needs to be verified through further clinical applications. The phantom used in the present study was designed based on a $70 \mathrm{~kg}$ adult male. Therefore, further studies are needed to determine whether this is suitable for populations with other body types. Second, in the present study, a CT scanner and its supporting software were used to scan and measure the simulated pulmonary nodules. Therefore, further verification is needed to determine whether this is suitable for other types of CT scanners and computer-aided design software. Third, in the present study, the diameters of the simulated pulmonary nodules were $3,5,8$, and $10 \mathrm{~mm}$, respectively. Although these simulated the solid nodules and ground-glass nodules with the CT attenuation values of $100 \mathrm{HU}$ and $-800 \mathrm{HU}$ (tube voltage: $120 \mathrm{kVp}$ ), these could not completely simulate the pulmonary nodules encountered in clinical work, and there were great differences in size, shape, CT attenuation value, and other aspects [6,17-19]. Therefore, further in-depth studies are needed to verify the conclusions of the present study. Finally, in the present study, the detection rate of small nodules in different combinations of scanning conditions and different doses was not analyzed. Hence, further follow-up studies are needed.

\section{Conclusion}

In lung cancer screening, CT parameters should be as follows: tube voltage is more than $80 \mathrm{kVp}$, and tube current is $80 \mathrm{mAs}$, in order to meet the requirements for the accurate measurement of the diameter and volume of pulmonary nodules.

\section{Abbreviations \\ $C T$ : The computed tomography; Lung-RADS: The lung Reporting and Data System; NCCN: The National Comprehensive Cancer Network; NLST: The national lung screening test; ASIR: The adaptive statistical iterative reconstruction; $C T_{D I}$ vol: $C T$ dose index; DLP: Dose length product; $E D$ : EFFE CTIVE dose; LSD: The least significant difference; SNR: The signal-to-noise ratio}

\section{Acknowledgements}

We would like to acknowledge the hard and dedicated work of all the staff that implemented the intervention and evaluation components of the study.

\section{Authors' contributions}

YD and GS conceived the idea and conceptualised the study. YW and QW collected the data. HF analysed the data. YD and GS drafted the manuscript, then QW and HF reviewed the manuscript. All authors read and approved the final draft. The author(s) declare(s) that they had full access to all of the data in this study and the author(s) take(s) complete responsibility for the integrity of the data and the accuracy of the data analysis.

\section{Funding}

No external funding received to conduct this study.

\section{Availability of data and materials}

The datasets used and/or analysed during the current study available from the corresponding author on reasonable request.

\section{Ethics approval and consent to participate}

I confirm that I have read the Editorial Policy pages. This study was conducted with approval from the Ethics Committee of the Fourth Hospital of Hebei Medical University. This study was conducted in accordance with the declaration of Helsinki. Consent is not applicable as there were no human participants.

\section{Consent for publication}

Not applicable.

Competing interests

All the authors declare that they have no competing interest. 
Received: 11 June 2020 Accepted: 14 September 2020

Published online: 02 October 2020

\section{References}

1. Huang P, Park S, Yan R, et al. Added value of computer-aided CT image features for early lung cancer diagnosis with small pulmonary nodules: a matched case-control study. Radiology. 2018;286:286-95.

2. MacMahon H, Austin JH, Gamsu G, Fleischner Society, et al. Guidelines for management of small pulmonary nodules detected on CT scans: a statement from the Fleischner society. Radiology. 2005;237:395-400.

3. Callister ME, Baldwin DR, Akram AR, British Thoracic Society Pulmonary Nodule Guideline Development Group; British Thoracic Society Standards of Care Committee, et al. British Thoracic Society guidelines for the investigation and management of pulmonary nodules. Thorax. 2015; 70(Suppl 2):ii1-ii54.

4. MacMahon H, Naidich DP, Goo JM, et al. Guidelines for management of incidental pulmonary nodules detected on CT images: from the Fleischner society 2017. Radiology. 2017;284:228-43.

5. Horeweg N, van Rosmalen J, Heuvelmans MA, et al. Lung cancer probability in patients with CT-detected pulmonary nodules: a prespecified analysis of data from the NELSON trial of low-dose CT screening. Lancet Oncol. 2014; 15:1332-41.

6. Clark TJ, Flood TF, Maximin ST, et al. Lung CT screening reporting and data system speed and accuracy are increased with the use of a Semiautomated computer application. J Am Coll Radiol. 2015;12(12 Pt A):1301-6.

7. Wood DE, Kazerooni EA, Baum SL, et al. Lung cancer screening, version 3 . 2018, NCCN clinical practice guidelines in oncology. J Natl Compr Cancer Netw. 2018;16:412-41.

8. Kim J, Lee KH, Kim J, et al. Improved repeatability of subsolid nodule measurement in low-dose lung screening with monoenergetic images: a phantom study. Quant Imaging Med Surg. 2019;9:171-9.

9. National Lung Screening Trial Research Team, Aberle DR, Adams AM, Berg $C D$, et al. Reduced lung-cancer mortality with low-dose computed tomographic screening. N Engl J Med. 2011;365:395-409.

10. National Lung Screening Trial Research Team, Church TR, Black WC, Aberle DR, et al. Results of initial low-dose computed tomographic screening for lung cancer. N Engl J Med. 2013;368:1980-91.

11. Christe $A$, Lin MC, Yen $A C$, et al. CT patterns of fungal pulmonary infections of the lung: comparison of standard-dose and simulated low-dose CT. Eur J Radiol. 2012:81:2860-6.

12. Carey $N$, Miller $F$, Bunn L. Developing a radiograher reporting service for $C T$ lung nodule surveillance. Lung Cancer. 2018;115:S24.

13. Christe A, Charimo-Torrente J, Roychoudhury K, et al. Accuracy of low-dose computed tomography $(\mathrm{CT})$ for detecting and character-izing the most common CT-patterns of pulmonary disease. Eur J Radiol. 2013;82:e142-50.

14. Szucs-Farkas Z, Schibler F, Cullmann J, et al. Diagnostic accuracy of pulmonary CT angiography at low tube voltage: Intraindividual comparison of a Normal-dose protocol at $120 \mathrm{kVp}$ and a low-dose protocol at $80 \mathrm{kVp}$ using reduced amount of contrast medium in a simulation study. AJR Am J Roentgenol. 2011;197:W852-9.

15. Schueller-Weidekamm C, Schaefer-Prokop CM, Weber M, et al. CT angiography of pulmonary arteries to detect pulmonary embolism: improvement of vascular enhancement with low kilovoltage settings. Radiology. 2006;241:899-907.

16. Gould MK, Donington J, Lynch WR, et al. Evaluation of individuals with pulmonary nodules: when is it lung cancer? Diagnosis and management of lung cancer, 3rd ed: American College of Chest Physicians evidence-based clinical practice guidelines. Chest. 2013;143(5 Suppl):e93S-e120S.

17. Ko JP, Suh J, Ibidapo O, et al. Lung adenocarcinoma: correlation of quantitative CT findings with pathologic findings. Radiology. 2016;280: 931-9.

18. Chung $\mathrm{K}$, Jacobs $\mathrm{C}$, Scholten ET, et al. Lung-RADS category $4 \mathrm{X}$ : does it improve prediction of malignancy in subsolid nodules? Radiology. 2017;284: 264-71

19. Ruilong Z, Daohai X, Li G, et al. Diagnostic value of 18F-FDG-PET/CT for the evaluation of solitary pulmonary nodules: a systematic review and metaanalysis. Nucl Med Commun. 2017;38:67-75.

\section{Publisher's Note}

Springer Nature remains neutral with regard to jurisdictional claims in published maps and institutional affiliations.

Ready to submit your research? Choose BMC and benefit from:

- fast, convenient online submission

- thorough peer review by experienced researchers in your field

- rapid publication on acceptance

- support for research data, including large and complex data types

- gold Open Access which fosters wider collaboration and increased citations

- maximum visibility for your research: over $100 \mathrm{M}$ website views per year

At $\mathrm{BMC}$, research is always in progress.

Learn more biomedcentral.com/submissions 